\title{
Real-world Surgical Outcomes of Primary Angle-closure Glaucoma
}

\author{
Boonsong \\ Wanichwecharungruang (iD) 1,2 \\ Chompunoot Phumratprapin 1,3 \\ Kittipong Kongsomboon ${ }^{4}$ \\ Kasem Seresirikachorn' \\ 'Glaucoma Services, Department of \\ Ophthalmology, Rajavithi Hospital and \\ Rangsit Medical College, Bangkok, \\ Thailand; ${ }^{2}$ Department of \\ Ophthalmology, Priest Hospital, Bangkok, \\ Thailand; ${ }^{3}$ Department of \\ Ophthalmology, Hua Chiew Hospital, \\ Bangkok, Thailand; ${ }^{4}$ Department of \\ Preventive and Social Medicine, Faculty of \\ Medicine, Srinakharinwirot University, \\ Nakhon Nayok, Thailand
}

Purpose: To evaluate surgical outcomes of the four common procedures utilized for primary angle-closure glaucoma (PACG).

Methods: A retrospective study of survival rate in surgical management of PACG was conducted in a referral eye center. One hundred and ninety-nine eyes from 173 PACG patients were collected for chart review. The procedures used were phacoemulsification (PE), combined PE with goniosynechialysis (PE-GSL), combined PE with trabeculectomy (PE-Trab), and trabeculectomy alone. Failure was defined as postoperative IOP $>21 \mathrm{mmHg}$ in patients who needed second surgical intervention or those who had IOP $<5 \mathrm{mmHg}$ with loss of light perception. Cumulative survival rates, risk of surgical failure, and complications were analyzed.

Results: PE, PE-GSL, PE-Trab, and trabeculectomy were performed in 84 eyes (42.2\%), 76 eyes $(38.2 \%), 21$ eyes $(10.6 \%)$, and 18 eyes $(9 \%)$, respectively. Cumulative survival rates at 60 months were $13 \%, 55 \%, 42 \%$ and $43 \%$, respectively. Cox regression analysis indicated that each $\mathrm{mmHg}$ IOP increased, the risk of surgical failure decreased by $13 \%$ (adjusted hazard ratio (HR) 0.87 ; 95\%CI: $0.84-0.93, p<0.001$ ).

Conclusion: Real-world surgical outcomes of PACG showed that PE alone had a low survival rate of $13 \%$ in 60 -month follow-up whereas PE-GSL achieved the highest rate of $55 \%$. PE-GSL should be initially considered for management of PACG, since it can restore and sustain the physiologic aqueous pathway and preserve the conjunctiva for future filtering surgery if needed.

Keywords: primary angle-closure glaucoma, surgical outcome, combined procedure, phacoemulsification, trabeculectomy, goniosynechialysis, survival, Asian, complication

\section{Introduction}

Primary angle-closure glaucoma (PACG) is a leading cause of blindness in Asia, and a meta-analysis of global glaucoma has demonstrated that the prevalence of PACG is highest in Asians. ${ }^{1}$ This is an anatomic disorder ${ }^{2}$ which usually occurs in a relatively small eye with crowded anterior segment, leading to iridotrabecular contact and resulting in intraocular pressure (IOP) elevation. ${ }^{3}$ Relative pupillary block is a common mechanism of PACG, and its definitive treatment is laser peripheral iridotomy (LPI); however, mechanisms other than pupillary block may be involved in PACG. In the Liwan Eye Study, one-fifth of primary angle-closure suspects (PACS) still had residual angle closure after $\mathrm{LPI}^{4}$ while Yan et al reported that two-thirds of Chinese cases of PACG had appositional angle closure after laser treatment..$^{5}$ Additional mechanisms, eg plateau iris, ${ }^{6,7}$ enlarged or anteriorly positioned lens, support the peripheral iris, leading to peripheral anterior synechia
Correspondence: Boonsong Wanichwecharungruang Glaucoma Services, Department of Ophthalmology, Rajavithi Hospital and Rangsit Medical College, 2 Phayathai

Road, Bangkok, 10400, Thailand

Tel +66 8I 3135040

Email boonsongw@yahoo.com 
(PAS) formation, and persistent IOP elevation will develop unless PAS is managed in a timely manner. Argon laser peripheral iridoplasty (ALPI) is an alternative treatment for primary-angle closure (PAC) $)^{8,9}$ and has been found to achieve successful long-term outcomes for plateau iris syndrome. ${ }^{10}$ In addition, EAGLE study demonstrated that clear lens extraction had better outcome than LPI in treatment of PACG. ${ }^{11}$

Surgical interventions can be applied to control the IOP of PACG when laser and medical treatment have failed. The results of phacoemulsification (PE) alone, combined PE with goniosynechialysis (PE-GSL), combined PE and trabeculectomy (PE-Trab), and trabeculectomy alone have been reported by various study groups, but the outcomes are somewhat difficult to compare because of the differences in definitions used in the reports. Herein, we review the charts of PACG patients who underwent the four procedures in our eye center and reported their outcomes, and it is representative of real-world practice for PACG patients who required surgical intervention.

\section{Patients and Methods}

This study was a retrospective chart review of PACG patients who underwent surgical intervention between August 2000 and December 2014. All investigations were carried out in accordance with the Declaration of Helsinki. The study protocol was approved by the Institutional Review Board of Rajavithi Hospital in May 2015, document No. 112/2558. Informed consent was waived and data was analyzed anonymously.

Inclusion criteria were patients who were $\geq 40$ years old, phakic, and had no previous intraocular surgery. All patients were diagnosed as PAC and PACG. PAC was defined as those who had trabecular meshwork apposition or closed at least 180 degrees obtained with gonioscopy, with IOP $>21 \mathrm{mmHg}$ or with PAS. And PACG defined as PAC with glaucomatous optic nerve and visual field damage. Acute angle-closure glaucoma (AACG) was defined as present when there were symptoms and signs of suddenly increased IOP, such as ocular pain, halo, corneal edema, and IOP $\geq 21 \mathrm{mmHg}$. Chronic angle-closure glaucoma (CACG) was defined as an asymptomatic form of PACG and was taken as present in patients who had onset of AACG $>6$ months.

Patients underwent LPI and/or ALPI, and glaucoma medications were prescribed if the IOP was uncontrolled. Indications for surgical intervention included:
1. Uncontrolled IOP despite maximally tolerated medical therapy

2. PACG with visually significant cataract

3. Poor compliance with medical treatment.

Ocular biometry, including axial length and intraocular lens (IOL) power, was obtained with IOL Master 500 (Carl Zeiss Meditec, CA, USA). If IOL Master could not obtain measurements from dense cataract, A-scan ultrasound, OcuScan (Alcon Laboratories, Fort Worth, TX, USA) was employed.

\section{Surgical Interventions}

The four procedures used for PACG in our eye center were performed under topical or local anesthesia and are briefly described below.

1. PE alone: temporal clear cornea PE with posterior chamber IOL (PC IOL) insertion was performed.

2. Combined PE-GSL: PE-IOL was first made, after which GSL was performed with cyclo-spatula under direct visualized Swan-Jacob goniolens, in all of the PAS area.

3. Combined PE-Trab: PE-IOL was first made, followed by superior fornix-based trabeculectomy with mitomycin C $(0.4 \mathrm{mg} / \mathrm{mL})$, with four-minute subconjunctival application.

4. Trabeculectomy alone: superior fornix-based trabeculectomy, with mitomycin $\mathrm{C}(0.4 \mathrm{mg} / \mathrm{mL})$ fourminute application, was performed.

The choice of procedure for each patient was based on individual clinical settings, and decisions were made by a senior glaucoma specialist (BW). The procedure was performed by the instructor (BW) and a number of clinical fellows under his supervision. Intra-, early- and late-postoperative complications were recorded.

Postoperative medications included prednisolone acetate $1 \%$, and antibiotic (tobramycin or levofloxacin) eye drops qid for four weeks after which the prednisolone acetate was tapered off gradually in the following four weeks. Postoperative data were collected for analysis at one month, three months, six months, 12 months, and then every six months until 60-month follow-up. Additional glaucoma medication and follow-up was made as needed. 


\section{Definition of Surgical Failure}

Surgical failure was defined as present in patients with postoperative IOP $>21 \mathrm{mmHg}$ with medical treatment at least two visits, and needed second surgical intervention (SSI), eg revised trabeculectomy or glaucoma drainage device. Those who had IOP $<5 \mathrm{mmHg}$ with loss of light perception were also classified as failure. Needling bleb revision was not defined as SSI or surgical failure. ${ }^{12}$

\section{Data Analyses}

Data was recorded with SPSS 16.0 (SPSS Inc., Chicago IL, USA), and statistical analyses were performed to compare the outcomes of the four surgical procedures. Subgroup analysis of the results of AACG and CACG was also performed, and distributions of the data were tested using the Kolmogorov-Smirnov test. Time to failure was analyzed with Kaplan-Meier estimates, and comparison among the procedures was made first with log rank test and then with Cox regression analysis. We used Mann-Whitney $U$-test for nonparametric data analysis, and Student's $t$-test and ANOVA were employed for continuous variables. Significance was set at $p<0.05$.

\section{Results}

The records of 188 patients were retrieved from hospital data relating to surgical treatment of PACG. Fifteen patients were excluded because they were lost to followup during the first three months postoperatively, and 199 eyes were included from 173 patients of whom 123 $(71.1 \%)$ were female, and 50 (28.9\%) were male. Table 1 shows various demographic data, including age, sex, underlying disease, family history of glaucoma, diagnosis, type of surgery, preoperative best-corrected visual acuity, IOP, number of glaucoma medications, axial length and cup to disc ratio (C/D). Median age at the time of the operations was 67 years (IQR 58-71), median preoperative IOP was $19 \mathrm{mmHg}$ (IQR 15-27), and median number of medications was three (IQR 2-4).

In Table 2, a total of 84 (42.2\%) eyes underwent PE alone, 76 (38.2\%) had PE-GSL, 21 (10.6\%) received PETrab, and 18 (9\%) had trabeculectomy. PE had the highest

Table I Patient Characteristic and Demographic Data (Number of Patients=173)

\begin{tabular}{|c|c|}
\hline Number of Eyes (\%) & $199(100)$ \\
\hline \multicolumn{2}{|l|}{ Gender } \\
\hline Male (\%) & $59(29.6)$ \\
\hline Female (\%) & $140(70.4)$ \\
\hline Age, median (interquartile range) years & $67(58-7 I)$ \\
\hline \multicolumn{2}{|l|}{ Underlying disease } \\
\hline Hypertension (\%) & $91(45.7)$ \\
\hline Diabetic mellitus (\%) & $38(19.1)$ \\
\hline Dyslipidemia (\%) & $38(19.1)$ \\
\hline Family history of glaucoma & $5(2.5)$ \\
\hline \multicolumn{2}{|l|}{ Diagnosis } \\
\hline AACC (\%) & $50(25.1)$ \\
\hline CACG (\%) & 149 (74.9) \\
\hline \multicolumn{2}{|l|}{ Procedure } \\
\hline PE (\%) & $84(42.2)$ \\
\hline PE-GSL (\%) & $76(38.2)$ \\
\hline PE-Trab (\%) & $21(10.6)$ \\
\hline Trab (\%) & $18(9)$ \\
\hline Preoperative IOP, median (interquartile range) $\mathrm{mmHg}$ & $19(15-27)$ \\
\hline Number of medication, median (interquartile range) & $3(2-4)$ \\
\hline Cup to disc ratio, median (interquartile range) & $0.8(0.5-0.9)$ \\
\hline Axial length, median (interquartile range) $\mathrm{mm}$ & $22.65(21.95-23.15)$ \\
\hline
\end{tabular}




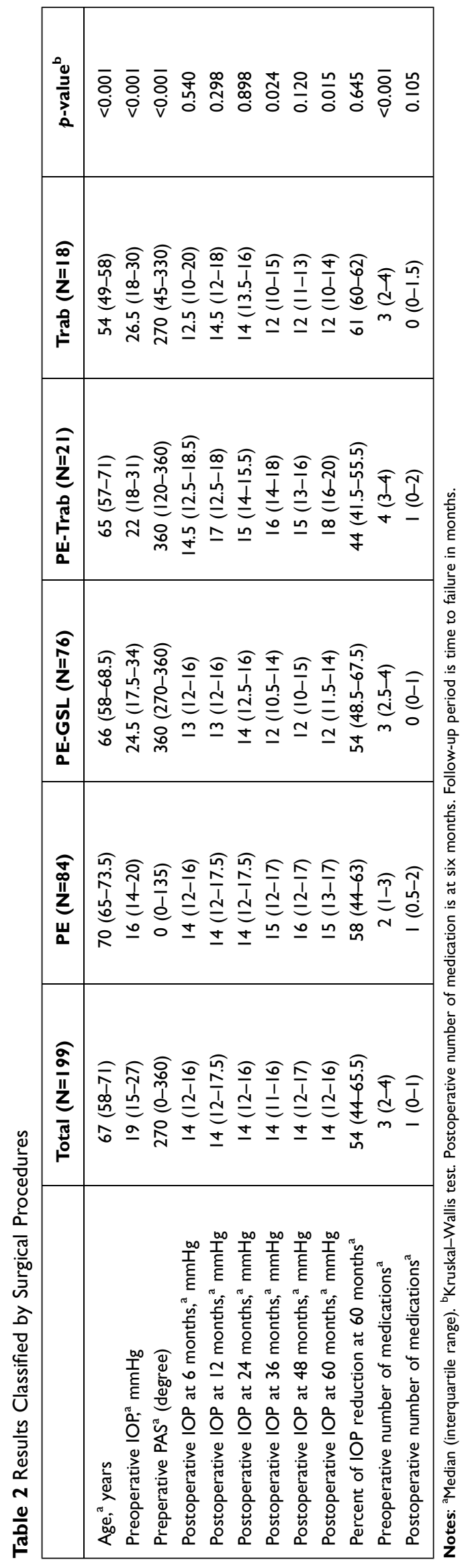

median patient age, and trabeculectomy had the lowest (70, 66, 65, and 54 years respectively, $p<0.001$ ). Median IOP reduction was 54\% (IQR 44-65.5), and the final number of medications decreased to one (IQR $0-1$ ). Median IOP reduction in was highest in Trab and lowest in PE-Trab.

Median pre- and postoperative IOP ( $\mathrm{mmHg}$ ) classified by surgical procedures are shown in Figure 1, presenting that IOP significantly decreased from six months onwards.

Median survival of all procedures was 24 months while cumulative survival of overall procedures at the 60-month follow-up was 0.26 , Figure 2.

Median survival time of PE alone, PE-GSL, PE-Trab, and trabeculectomy were $6,60,12$, and 48 months respectively, and cumulative survival rates at 60 months were $13 \%, 55 \%, 42 \%$ and $43 \%$ respectively, Figure 3 .

Cox regression analysis indicated that higher preoperative IOP was a protective factor of the surgical outcome. When we adjusted the regression model with covariates of age, sex, diabetes mellitus, numbers of medication, and axial length, the adjusted HR was 0.87 ; $95 \%$ CI: $0.84-0.93$, $p<0.001$. In plain words, each mmHg of IOP increased, the chance of failure decreased by $13 \%$. Preoperative PAS was not related to surgical failure of these procedures (Table 3).

Subgroups of AACG (50 eyes) and CACG (149 eyes) were analyzed. We performed PE-GSL in $70 \%$ of AACG and PE in $49 \%$ of CACG, Table 4 . Mean IOP reductions in the groups were $51.5 \%$ (SD 18.25) and 55.06\% (SD 13.92) respectively at the 60-month follow-up (Table 5).

Figure 4 demonstrated Kaplan-Meier plots of surgical failure between acute and chronic angle-closure glaucoma, with nonsignificant difference $(p=0.538, \log$ rank test).

\section{Complications and Second Surgical Intervention}

Perioperative complications, up to the three-month followup, were shown in Table 6. Most cases were self-limited or manageable. None of these patients experienced loss of vision to no light perception from hypotony. Vision-threatening events such as suprachoroidal hemorrhage or endophthalmitis was not found in this chart review from early to late follow-up. Two patients, one in PE-GSL and one in PE-Trab, developed epiretinal membrane in this study. 


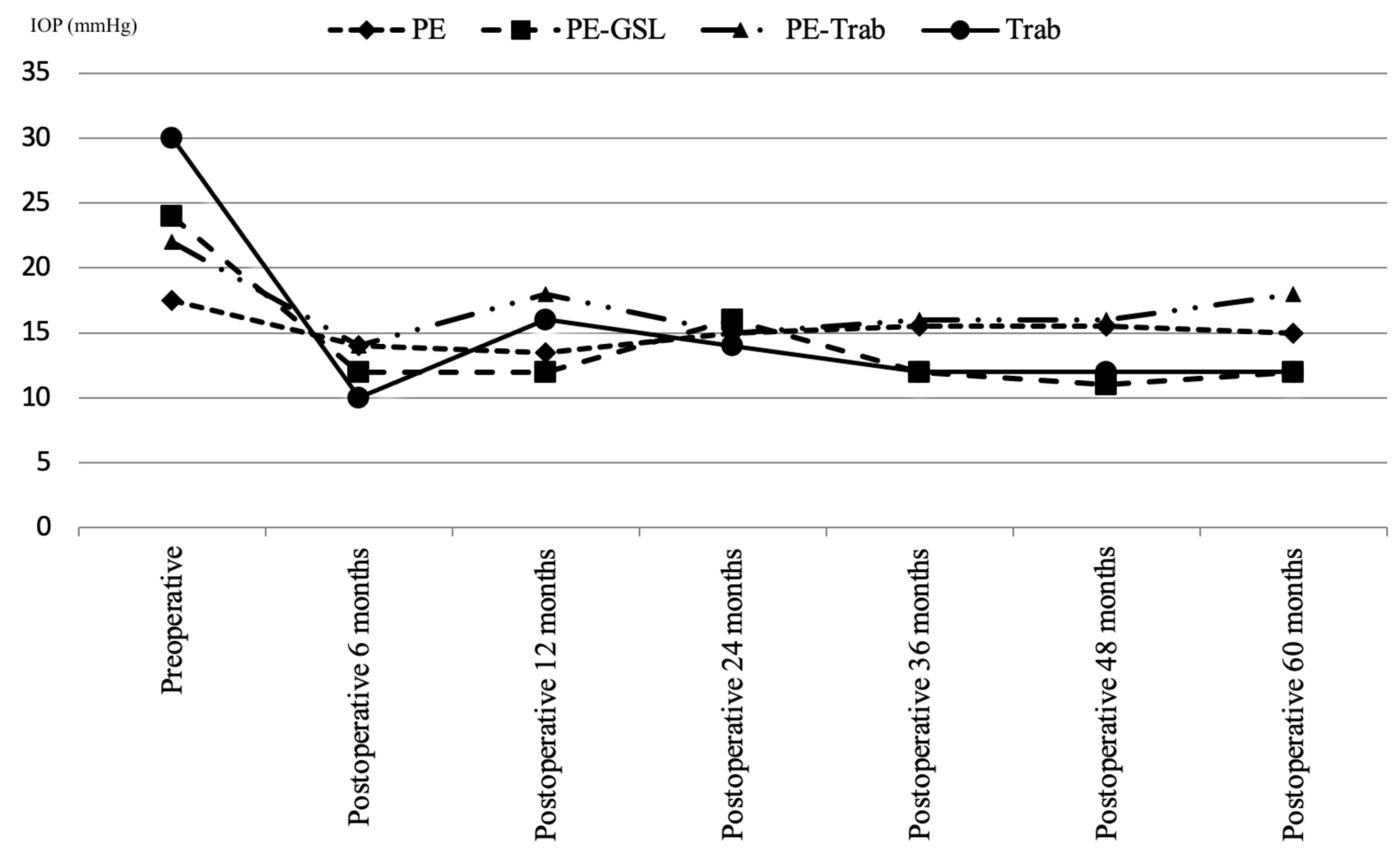

Figure I Median pre- and post-operative IOP $(\mathrm{mmHg})$ classified by surgical procedures. IOP significantly decreased from six months onwards.

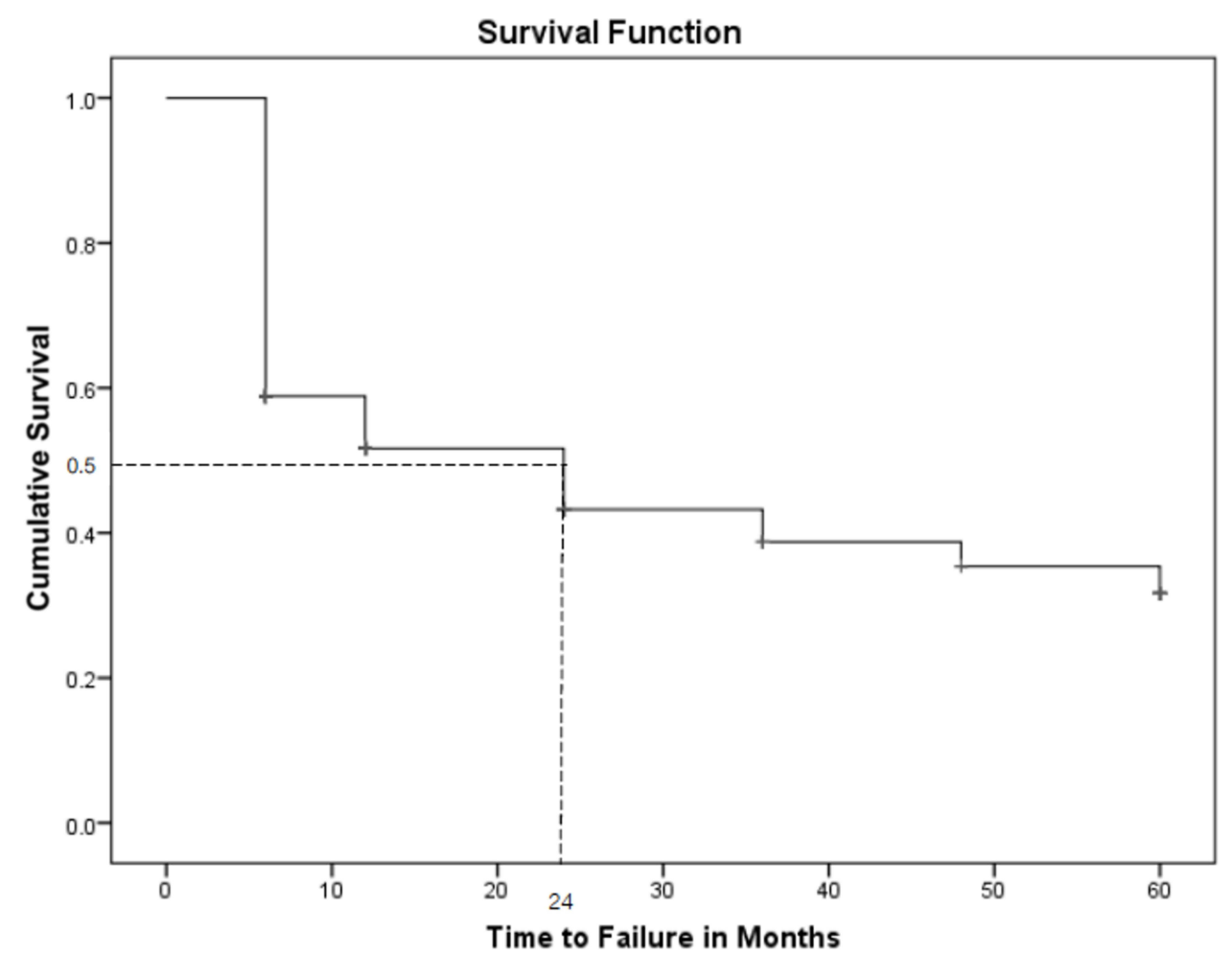

Figure 2 Kaplan-Meier estimates of surgical failure across the four procedures. 


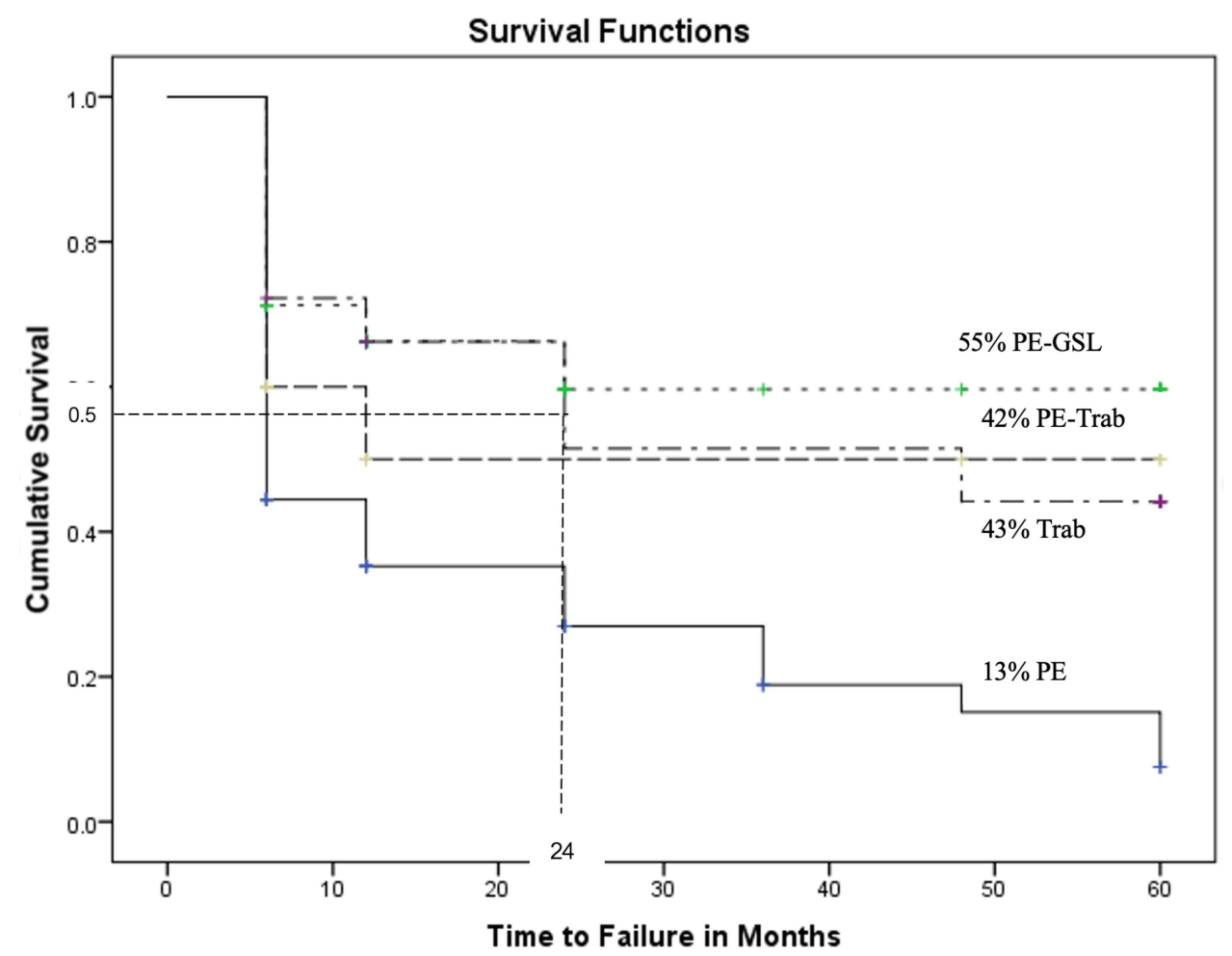

Figure 3 Median survival time of PE alone, PE-GSL, PE-Trab, and trabeculectomy were 6, 60, I2, and 48 months respectively, and cumulative survival rates at 60 months were $13 \%, 55 \%, 42 \%$ and $43 \%$ respectively.

The 14 eyes of 14 patients for whom the first procedure failed were managed as follows: six eyes after failed PE alone underwent trabeculectomy; two eyes after failed PE-GSL received trabeculectomy; two eyes that underwent unsuccessful combined PE-Trab underwent revised trabeculectomy; one eye of failed PE-Trab underwent Ahmed glaucoma valve; and patients who had failed trabeculectomy underwent PE (one eye), PE-GSL (one eye), and PE-Trab (one eye).

Table 3 Factors Related to Failure Time by Cox Regression

\begin{tabular}{|c|c|c|c|c|}
\hline \multicolumn{2}{|l|}{ Factors } & Adjusted $\mathbf{H R}^{\mathrm{a}}$ & $95 \% \mathrm{Cl}$ & $p$-value \\
\hline \multicolumn{2}{|c|}{ Preperative IOP } & 0.87 & $0.84-0.93$ & $<0.001$ \\
\hline \multicolumn{2}{|l|}{ PAS } & 1 & $0.99-1.01$ & 0.834 \\
\hline \multicolumn{2}{|c|}{ Numbers of medication } & 1.10 & $0.87-1.39$ & 0.421 \\
\hline \multicolumn{2}{|l|}{ Axial length } & I.0I & $0.73-1.40$ & 0.938 \\
\hline \multicolumn{2}{|l|}{ Age } & 1.01 & $0.98-1.05$ & 0.480 \\
\hline Sex & $\begin{array}{l}\text { Male } \\
\text { Female }\end{array}$ & $\begin{array}{c}1 \\
1.17\end{array}$ & $\begin{array}{c}- \\
0.66-2.07\end{array}$ & $\begin{array}{c}- \\
0.588\end{array}$ \\
\hline Diabetes & $\begin{array}{l}\text { No } \\
\text { Yes }\end{array}$ & $\begin{array}{c}1 \\
1.36\end{array}$ & $\begin{array}{c}- \\
0.77-2.41\end{array}$ & $\begin{array}{c}- \\
0.294\end{array}$ \\
\hline Procedure & $\begin{array}{l}\text { PE } \\
\text { PE-GSL } \\
\text { PE-Trab } \\
\text { Trab }\end{array}$ & $\begin{array}{c}1 \\
0.83 \\
0.81 \\
1.63\end{array}$ & $\begin{array}{c}- \\
0.43-1.59 \\
0.27-2.47 \\
0.34-7.72\end{array}$ & $\begin{array}{c}- \\
0.575 \\
0.712 \\
0.541\end{array}$ \\
\hline
\end{tabular}

Notes: ${ }^{a}$ Adjusted with age, sex, diabetes mellitus, numbers of medication, preoperative IOP, and axial length. 
Table 4 Surgical Procedures Between Acute and Chronic PACG

\begin{tabular}{|l|c|c|c|c|c|}
\hline & PE & PE-GSL & PE-Trab & Trab & Total \\
\hline AACG (\%) & II (22) & $35(70)$ & $3(6)$ & I (2) & $50(100)$ \\
CACG (\%) & $73(49)$ & $41(27.5)$ & $18(12.1)$ & $17(11.4)$ & $149(100)$ \\
\hline
\end{tabular}

Table 5 Surgical Outcomes Between Acute and Chronic Angle-closure Glaucoma

\begin{tabular}{|c|c|c|c|}
\hline & AACG $(N=50)$ & CACG $(N=149)$ & $p$-value \\
\hline Age, ${ }^{a}$ years & $63.5(57-68)$ & $67(58-72)$ & $0.008^{\mathrm{b}}$ \\
\hline Sex, Female (\%) & $40(80)$ & $100(67.1)$ & $0.084^{c}$ \\
\hline Axial length, ${ }^{\mathrm{a}}, \mathrm{mm}$ & $22.21(21.63-22.65)$ & $22.78(22.26-23.27)$ & $<0.001^{\mathrm{b}}$ \\
\hline Preoperative IOP, ${ }^{\mathrm{a}} \mathrm{mmHg}$ & $24(16-38)$ & $18(15-25)$ & $0.014^{\mathrm{b}}$ \\
\hline Postoperative IOP, ${ }^{\mathrm{a}} \mathrm{mmHg}$ & $12(11-15)$ & $14(1|-| 7)$ & $0.125^{\mathrm{b}}$ \\
\hline Percent of IOP reduction (mean \pm SD) & $51.50 \pm 18.25$ & $55.06 \pm 13.92$ & $0.573^{d}$ \\
\hline Preoperative number of medications $\mathrm{a}^{\mathrm{a}}$ & $3(2-4)$ & $3(2-4)$ & $0.075^{\mathrm{b}}$ \\
\hline Postoperative number of medications ${ }^{\mathrm{a}}$ & $0(0-1)$ & I $(0-2)$ & $0.27 I^{\mathrm{b}}$ \\
\hline
\end{tabular}

Notes: ${ }^{\mathrm{a}}$ Median (interquartile range). ${ }^{\mathrm{b}}$ Mann-Whitney U-test. ${ }^{\mathrm{C}} \mathrm{Chi}$-squared test. ${ }^{\mathrm{d}}$ Independent $t$-test.

\section{Discussion}

In the present study, the 60 -month survival rate of PE-GSL was the highest (55\%), followed by trabeculectomy alone (43\%), PE-Trab (42\%), and PE alone (13\%). Subgroup analysis of the survival rates between AACG and CACG showed no significant difference. Of interest, our report demonstrated that the higher preoperative IOP was related to the higher success rate of the procedures. There might be a few explanations for this finding. First, the surgeon might consider a surgical technique which could be more aggressive IOP lowering, for instance, extensive GSL, or made a larger internal ostium of trabeculectomy. Second, postoperative care might be more intensive. And third, the surgeon might consider early surgery in AACG which had

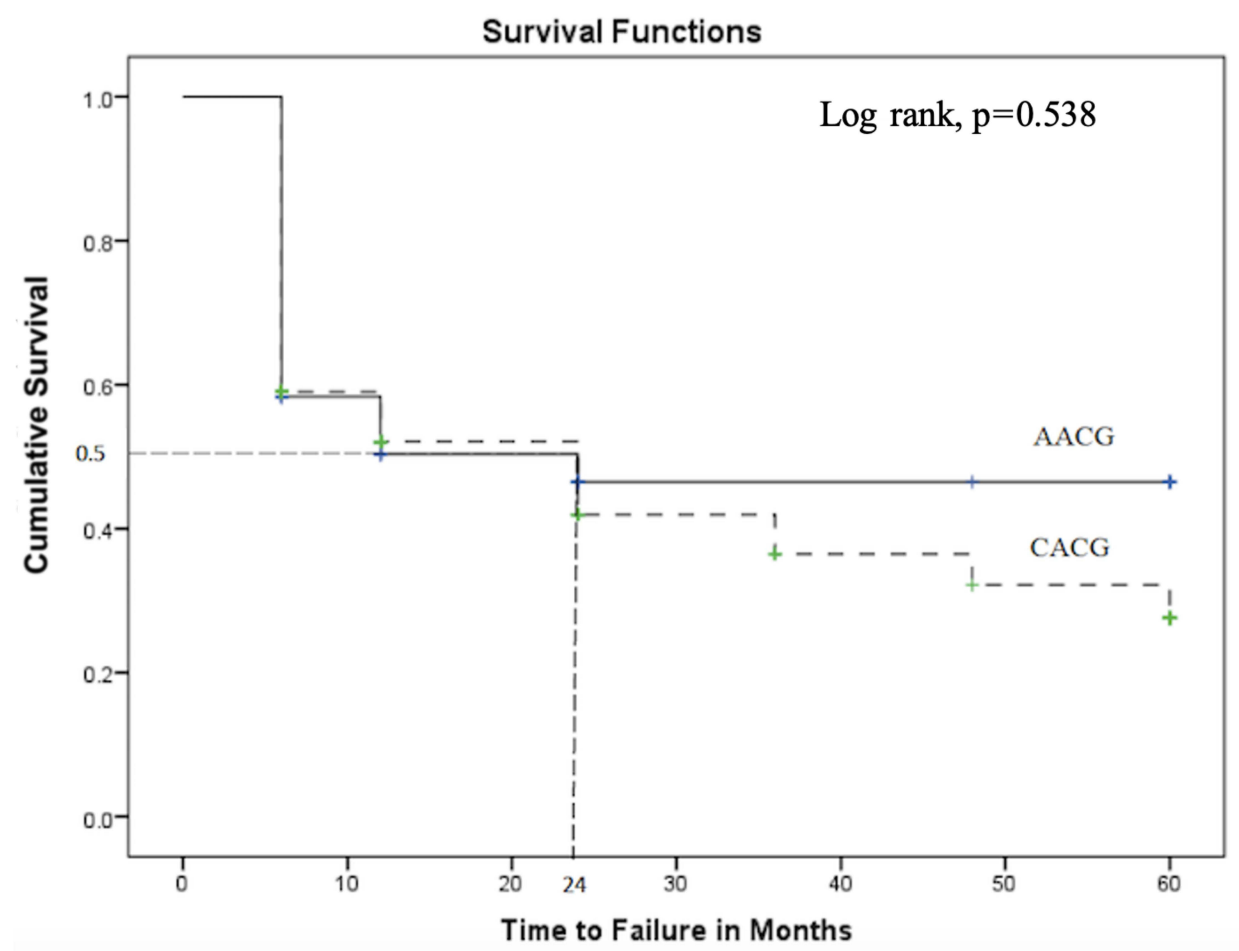

Figure 4 Kaplan-Meier plots of surgical failure between acute and chronic angle-closure glaucoma, with nonsignificant difference. 
Table 6 Perioperative Complications of the Procedures for PACG

\begin{tabular}{|l|l|l|l|l|}
\hline & PE (N=84) N (\%) & PE-GSL (N=76) N (\%) & PE-Trab (N=2 I) N (\%) & Trab (N= I 8) N (\%) \\
\hline Shallow AC & - & - & $5(23.8)$ & $3(16.7)$ \\
AC bleeding & I (I.2) & $8(I 0.4)$ & $2(9.5)$ & $2(I I . I)$ \\
Fibrinoid AC reaction & - & $2(2.6)$ & I (4.8) & - \\
Inflammation >3 months & $3(3.6)$ & $2(2.6)$ & I (4.8) & I (5.6) \\
Wound leak & - & - & $4(19)$ & I (5.6) \\
Hypotony maculopathy & - & - & I (4.8) & I (5.6) \\
Choroidal detachment & - & I (I.3) & $4(4.8)$ & $2(I I . I)$ \\
Encapsulated bleb & - & - & $5(23.8)$ & $2(I I . I)$ \\
Needling revision & - & - & I (5.6) \\
\hline
\end{tabular}

a higher IOP, this could prevent subsequent trabecular damage from PAS formation.

Surgical management for PACG is evolving and still being debated, ${ }^{13}$ and although comparative studies between two procedures have been reported by various authors (Table 7), real-world practice is sparsely reported.

PACG appears to involve multiple factors, including pupillary block, plateau iris, lens and posterior to lens mechanisms. ${ }^{3}$ Proper surgical management for individual patients may save the eye from glaucoma's blinding process.

For decades, trabeculectomy has been the standard procedure for most cases of glaucoma; however, its success rate is rather low, particularly in AACG. It has been reported to have a success rate of $56 \%$ in medical uncontrolled AACG; ${ }^{8}$ however, PE has shown good outcomes for $\mathrm{AACG}^{20}$ It is common to find coexisting glaucoma and cataracts in elderly patients. The lens component of PACG is caused by thick lens and/or anteriorly positioned lens, and choice of clinical treatment for patients who have both conditions is based on the general principles of glaucoma and cataract surgery. If glaucoma (IOP elevation) is more prominent, trabeculectomy should be used, whereas in the opposite case, cataract surgery should be performed. If both glaucoma and cataract are prominent, combined glaucoma and cataract surgery is the preferred choice. Combined operations for PACG include PE-Trab and PE-GSL.

Goniosynechialysis involves stripping off the adhesion of the peripheral iris from the trabecular surface to restore the physiologic aqueous passage, ${ }^{21}$ this can reduce IOP in PACG if the function of the trabecular meshwork has not been damaged from long duration of PAS. We did not perform GSL alone because we were aware that the lens component would push the PAS back later. We always combine PE and GSL in our practice, as combined PEGSL has demonstrated a $90 \%$ success rate for PACG postacute attack in Asian patients. ${ }^{22}$ Favorable outcomes of PE-GSL have also been reported by some research groups ${ }^{23-25}$ however, a randomized controlled study by Husain et al reported that PE-GSL did not have any additional IOP-lowering effect compared to PE alone, ${ }^{19}$ and meta-analysis in seven randomized controlled trials also showed that PE-GSL was not superior to PE alone in terms of IOP reduction. ${ }^{26}$ Our data confirmed this, showing that median final IOP and percent IOP reduction were not significantly different between PE-GSL and PE alone.

Regarding combined PE-Trab in PACG, some randomized controlled trials have reported that combined PETrab demonstrated better IOP control than PE alone ${ }^{14}$

Table 7 Comparative Studies of Surgical Procedures for PACG

\begin{tabular}{|c|c|c|c|c|c|c|}
\hline Authors & Year & \multicolumn{2}{|c|}{ Surgical Interventions } & Number of Patients & Follow-up (Months) & Results \\
\hline Tham et $\mathrm{al}^{14}$ & 2009 & PE & PE-Trab & 27 vs 24 & 24 & PE-Trab lowered IOP better than PE \\
\hline Tsai et $\mathrm{al}^{12}$ & 2009 & Trab & PE-Trab & 24 vs 75 & 36 & NS \\
\hline Wang et $\mathrm{al}^{15}$ & 2012 & Trab & PE-Trab & 14 vs 17 & 10 & NS \\
\hline Tham et $\mathrm{al}^{16}$ & 2013 & $\mathrm{PE}$ & Trab & 26 vs 24 & 24 & NS \\
\hline Zhao et al ${ }^{17}$ & 2013 & PE-GSL & PE-Trab & 33 vs 32 & 12 & NS \\
\hline Song et al ${ }^{18}$ & 2016 & Trab & PE-Trab & 29 vs 15 & 12 & NS \\
\hline Husain et al ${ }^{19}$ & 2019 & $\mathrm{PE}$ & PE-GSL & 40 vs 38 & 12 & NS \\
\hline
\end{tabular}

Abbreviation: NS, not significantly different. 
while Zhang et al reported similar success rates with PE, trabeculectomy, and PE-Trab. ${ }^{27}$

Combined PE and glaucoma procedures, GSL or trabeculectomy, could yield some advantages in PACG. In addition to the lens removal effect in PACG, ${ }^{3}$ GSL can restore physiologic aqueous humor drainage, and trabeculectomy can bypass trabecular resistance. ${ }^{22,28}$ Zhao et al reported comparable effects of PE-GSL and PE-Trab in 65 Chinese eyes. ${ }^{17}$ They found that both procedures significantly lowered IOP from preoperative up to 12-month follow-up, and that PE-GSL was able to open the PAS ( $>270$ degrees) in all 18 eyes, so they concluded that PEGSL could be applied as primary surgery for PACG with coexisting cataract. In addition, the conjunctiva is preserved in PE-GSL, so that trabeculectomy could be performed if uncontrolled-IOP occurred subsequently.

Comparison of those results reveals limitations and may cause confusion. Firstly, they were conducted in various centers using diverse surgical techniques; for instance, Zhao et al performed GSL with a viscoelastic substance ${ }^{17}$ while we did so with a cyclo spatula; MMC dosage in trabeculectomy and combined PE-Trab was also different. In combined PE-Trab, Zhao et al did not apply MMC, ${ }^{17}$ whereas we used it as an adjunct in all cases. The definitions of success/failure were also different, and there is as yet no definite indication for these four procedures in PACG management.

In real-world clinical practice, the surgical decision to use PACG is based on many factors, such as preoperative IOP, number of medications, cataract status, lens thickening, anterior lens position, PAS, stage of glaucoma, and acute/chronic conditions. For example, we tend to perform PE alone in older patients who have quite well-controlled IOP, and we use trabeculectomy alone in younger patients with uncontrolled IOP. Median age of patients undergoing PE alone was 70 years, while that of trabeculectomy alone was 54 years, and median preoperative IOPs were 16 and $26.5 \mathrm{mmHg}$ respectively. In between those two groups, we chose combined PE-GSL or PE-Trab. Median ages of PE-GSL and PETrab patients were 66 vs 65 years, and preoperative IOPs were 24.5 and $22 \mathrm{mmHg}$ respectively. Combined PE-GSL was favored by our group for patients who had history of AACG (70\%) while combined PE-Trab was considered for those who might have a lower degree of PAS.

In the present study, PE alone had the lowest five-year survival at $13 \%$. Although it can eliminate pupillary block, deepen $\mathrm{ACD}$, and widen the peripheral angle, ${ }^{29-31} \mathrm{PE}$ alone may not eliminate preexisting PAS, and residual
PAS after PE could block aqueous outflow, resulting in IOP elevation. In addition, Sihota et al reported a histologic study of the trabecular meshwork of PACG, showing noninflammatory degeneration in AACG, and endothelial cell loss in CACG. ${ }^{32}$ Hamanaka et al reported Schlemm's canal occlusion with endothelial cell damage and fusion of the trabecular beam. ${ }^{33}$ Both trabecular and Schlemm's canal changes lead to IOP elevation after PE alone. In the EAGLE study, in contrast, clear lens extraction performed favorably in lowering IOP compared with laser iridotomy in three years of follow-up. ${ }^{11}$ The investigators employed PE in new cases of PAC and PACG with IOP $>30 \mathrm{mmHg}$ without cataract. We speculated that the aqueous drainage pathway might have been less changed in the EAGLE study than in ours. Early surgery might prevent PAS formation which can cause failure in the longterm follow-up period; however, there was markedly missing PAS data in the EAGLE study (247 from 419 patients). The chronic PACG in our patients might have had more damage to trabecular outflow than in the early glaucoma of the EAGLE study.

\section{Strengths and Limitations}

This study had both strengths and limitations. Its strengths include the fact that it used practice-based data, and the outcome measurements were analyzed within the same definitions and statistical methods. Long-term follow-up data is useful for validation of the outcomes of this chronic disease. Its limitations related to a retrospective manner of the study and included the fact that some data were missing. To date, there is no specific indication in various procedures for PACG yet. We made decisions arbitrarily and it could be one of the sources of bias. The numbers of patients in the groups and their follow-up periods were different, and the sample sizes were relatively small and varied among the groups. We had 26 patients who were operated on both sides, whereas 147 patients were operated on one side only. This could create a confounding factor, for the eyes would not be independent. This could therefore constitute a statistical bias.

In fact, trabeculectomy alone was uncommonly employed (9\%) because our patients were relatively old with cataractous formation, so cataract removal and combined procedures appeared to be a better choice for the status of these patients. Even though trabeculectomy proportion was less than $10 \%$, we did not exclude trabeculectomy from analysis in order to show a real-life practice for PACG. 
In this study we excluded patients who had complications such as ruptured PC and zonular dialysis, and this could have resulted in overestimation of the failure rates. Lens thickness could not be obtained with IOL Master 500, so that we could not clarify the effect of lens thickness in PACG surgery outcomes. Future research is needed to specify indication of these procedures for the specific type of PACG.

\section{Conclusions}

Real-world surgical outcomes of PACG showed that PE alone had a low survival rate of $13 \%$ in a 60 -month follow-up whereas PE-GSL achieved the highest rate of $55 \%$. PE-GSL should be initially considered for surgical management of PACG, since it can restore and sustain the physiologic aqueous drainage in a long-term follow-up period.

\section{Precis}

Real-world data showed that stand-alone phaco was not as effective as combined phaco-goniosynechialysis in management of PACG, which showed a five-year survival rate of $13 \%$ vs $55 \%$ respectively.

\section{Acknowledgments}

- We would like to thank John Flanagan, MA for language editing and proofreading the manuscript.

- Supported by Rajavithi Research Grant number 112/ 2558.

\section{Author Contributions}

All authors made substantial contributions to conception and design, acquisition of data, or analysis and interpretation of data; took part in drafting the article or revising it critically for important intellectual content; agreed to submit to the current journal; gave final approval of the version to be published; and agree to be accountable for all aspects of the work.

\section{Disclosure}

The authors reported no conflicts of interest in this work and have no conflict of interest to declare regarding any devices mentioned in the study.

\section{References}

1. Tham YC, Li X, Wong TY, Quigley HA, Aung T, Cheng CY. Global prevalence of glaucoma and projections of glaucoma burden through 2040: a systematic review and meta-analysis. Ophthalmology. 2014;121(11):2081-2090.
2. Ritch R, Lowe RF. Angle-closure glaucoma: mechanisms and epidemiology. In: Ritch R, Shields MB, Krupin T, editors. The Glaucomas. 2nd ed. St Louis: Mosby; 1996:801-819.

3. Ritch R, Liebmann J, Tello C. A construct for understanding angle of closure glaucoma: the role of ultrasound biomicroscopy. Ophthalmol Clin North Am. 1995;8:281-293.

4. He M, Friedman DS, Ge J, Huang W, Jin C, Lee PS, et al. Laser peripheral iridotomy in primary angle-closure suspects: biometric and gonioscopic outcomes: the Liwan Eye Study. Ophthalmology. 2007;114(3):494-500. doi:10.1016/j.ophtha.2006.06.053

5. Yan YJ, Wu LL, Wang X, Xiao GG. Appositional angle closure in Chinese with primary angle closure and primary angle closure glaucoma after laser peripheral iridotomy. Invest Ophthalmol Vis Sci. 2014;55(12):8506-8512.

6. Kumar RS, Tantisevi V, Wong MH, Laohapojanart K, Chansanti O, Quek DT, et al. Plateau iris in Asian subjects with primary angle closure glaucoma. Arch Ophthalmol. 2009;127(10):1269-1272.

7. Kumar G, Bali SJ, Panda A, Sobti A, Dada T. Prevalence of plateau iris configuration in primary angle closure glaucoma using ultrasound biomicroscopy in the Indian population. Indian $J$ Ophthalmol. 2012;60(3):175-178.

8. Lai JSM, Tham CCY, Chua JKH, Lam DSC. Immediate diode laser peripheral iridoplasty as treatment of acute attack of primary angle closure glaucoma: a preliminary study. J Glaucoma. 2001;10:89-94.

9. Lam DSC, Lai JSM, Tham CCY, Chua JKH, Poon ASY. Argon laser peripheral iridoplasty versus conventional systemic medical therapy in treatment of acute primary angle-closure glaucoma: a prospective, randomized, controlled trial. Ophthalmology. 2002;109(9):15911596.

10. Ritch R, Tham CC, Lam DS. Long-term success of argon laser peripheral iridoplasty in the management of plateau iris syndrome JID - 7802443. Ophthalmology. 2004;111(1):104-108.

11. Azuara-Blanco A, Burr J, Ramsay C, Cooper D, Foster PJ, Friedman DS, et al. Effectiveness of early lens extraction for the treatment of primary angle-closure glaucoma (EAGLE): a randomised controlled trial. Lancet. 2016;388(10052):1389-1397.

12. Tsai HY, Liu CJ, Cheng CY. Combined trabeculectomy and cataract extraction versus trabeculectomy alone in primary angle-closure glaucoma. Br J Ophthalmol. 2009;93(7):943-948.

13. Lai J, Choy BN, Shum JW. Management of primary angle-closure glaucoma. Asia Pac J Ophthalmol (Phila). 2016;5(1):59-62.

14. Tham CC, Kwong YY, Leung DY, Lam SW, Li FC, Chiu TY, et al. Phacoemulsification versus combined phacotrabeculectomy in medically uncontrolled chronic angle closure glaucoma with cataracts. Ophthalmology. 2009;116(4):725-31, 31 e1-3.

15. Wang M, Fang M, Bai Y, et al. Comparison of combined phacotrabeculectomy with trabeculectomy only in the treatment of primary angle-closure glaucoma. Chin Med J. 2012;125:1429-33

16. Tham CC, Kwong YY, Baig N, Leung DY, Li FC, Lam DS. Phacoemulsification versus trabeculectomy in medically uncontrolled chronic angle-closure glaucoma without cataract. Ophthalmology. 2013;120(1):62-7.

17. Zhao XJ, Yang XX, Fan YP, Li BH, Li Q. Comparison of combined phacoemulsification, intraocular lens implantation, and goniosynechialysis with phacotrabeculectomy in the treatment of primary angle-closure glaucoma and cataract. Asia Pac J Ophthalmol (Phila). 2013;2(5):286-290.

18. Song BJ, Ramanathan M, Morales E, Law SK, Giaconi JA, Coleman AL, et al. Trabeculectomy and Combined PhacoemulsificationTrabeculectomy: Outcomes and Risk Factors for Failure in Primary Angle Closure Glaucoma. J Glaucoma. 2016;25(9):763-9.

19. Husain R, Do T, Lai J, Kitnarong N, Nongpiur ME, Perera SA, et al. Efficacy of phacoemulsification alone vs phacoemulsification with goniosynechialysis in patients with primary angle-closure disease: a randomized clinical trial. JAMA Ophthalmol. 2019. 
20. Jacobi PC, Dietlein TS, Luke C, Engels B, Krieglstein GK. Primary phacoemulsification and intraocular lens implantation for acute angle-closure glaucoma. Ophthalmology. 2002;109(9):1597-1603.

21. Campbell DG, Vela A. Modern goniosynechialysis for the treatment of synechial angle-closure glaucoma. Ophthalmology. 1984;91 (9):1052-1060.

22. Teekhasaenee C, Ritch R. Combined phacoemulsification and goniosynechialysis for uncontrolled chronic angle-closure glaucoma after acute angle-closure glaucoma. Ophthalmology. 1999;106:669-675.

23. Lai JS, Tham CC, Lam DS. The efficacy and safety of combined phacoemulsification, intraocular lens implantation, and limited goniosynechialysis, followed by diode laser peripheral iridoplasty, in the treatment of cataract and chronic angle-closure glaucoma. J Glaucoma. 2001;10 (4):309-315.

24. Kameda $T$, Inoue $T$, Inatani $M$, Tanihara $H$; Japanese PhacoGoniosynechialysis Multicenter Study G. Long-term efficacy of goniosynechialysis combined with phacoemulsification for primary angle closure. Graefes Arch Clin Exp Ophthalmol. 2013;251(3):825-830.

25. White AJ, Orros JM, Healey PR. Outcomes of combined lens extraction and goniosynechialysis in angle closure. Clin Experiment Ophthalmol. 2013;41(8):746-752.

26. Wang N, Jia SB. Phacoemulsification with or without goniosynechialysis for angle-closure glaucoma: a global meta-analysis based on randomized controlled trials. Int J Ophthalmol. 2019;12(5):826-833.

27. Zhang XTL, Li A, Du S, Zhu Y, Ge J. The clinical outcomes of three surgical managements on primary angle-closure glaucoma. Yan Ke Хие Bao. 2007;23(2):65-74.
28. Tian T, Li M, Pan Y, Cai Y, Fang Y. The effect of phacoemulsification plus goniosynechialysis in acute and chronic angle closure patients with extensive goniosynechiae. BMC Ophthalmol. 2019;19(1):65.

29. Pereira FA, Cronemberger S. Ultrasound biomicroscopic study of anterior segment changes after phacoemulsification and foldable intraocular lens implantation. Ophthalmology. 2003;110(9):17991806.

30. Hayashi K, Hayashi H, Nakao F, Hayashi F. Changes in anterior chamber angle width and depth after intraocular lens implantation in eyes with glaucoma. Ophthalmology. 2000;107:698-703.

31. Hayashi K, Hayashi H, Nakao F, Hayashi F. Effect of cataract surgery on intraocular pressure control in glaucoma patients. $J$ Cataract Refract Surg. 2001;27(11):1779-1786.

32. Sihota R, Lakshmaiah NC, Walia KB, Sharma S, Pailoor J, Agarwal HC. The trabecular meshwork in acute and chronic angle closure glaucoma. Indian J Ophthalmol. 2001;49(4):255-259.

33. Hamanaka T, Kasahara K, Takemura T. Histopathology of the trabecular meshwork and Schlemm's canal in primary angle-closure glaucoma. Invest Ophthalmol Vis Sci. 2011;52(12):8849-8861.
Clinical Ophthalmology

\section{Publish your work in this journal}

Clinical Ophthalmology is an international, peer-reviewed journal covering all subspecialties within ophthalmology. Key topics include: Optometry; Visual science; Pharmacology and drug therapy in eye diseases; Basic Sciences; Primary and Secondary eye care; Patient Safety and Quality of Care Improvements. This journal is indexed on PubMed
Dovepress

Central and CAS, and is the official journal of The Society of Clinical Ophthalmology (SCO). The manuscript management system is completely online and includes a very quick and fair peer-review system, which is all easy to use. Visit http://www.dovepress.com/ testimonials.php to read real quotes from published authors. 